\title{
Evaluación diagnóstica del grado de adaptación de la ciudad de Castellón a las personas mayores
}

\author{
SHEILA EXPÓSITO MIRALLES \\ al262381@uji.es \\ JOAQUÍN MARTÍNEZ SÁNCHEZ \\ al128827@uji.es \\ Adrián Daniel Munteanu Munteanu \\ al225857@uji.es \\ Antonio Caballer Miedes \\ caballer@uji.es
}

\section{Resumen}

Introducción: Según la Organización Mundial de la Salud la población mundial ha sufrido, en los últimos años, un envejecimiento significativo (OMS, 2015). En el año 2050 el porcentaje de población mayor de 60 años se habrá duplicado, provocando que por primera vez en la historia la pirámide mundial poblacional se invierta. Además, en los últimos años, se ha producido una migración de la población mayor de 60 años desde las zonas rurales a los centros urbanos. Para el año 2030 se prevé que tres de cada cinco personas del mundo vivan en alguna ciudad y el número de habitantes urbanos en las regiones menos desarrolladas sea casi cuatro veces mayor que en las regiones desarrolladas. Por estos motivos, la ciudad de Castellón decide participar en el proyecto "Red mundial de ciudades amigables con las personas mayores» promovido por la OMS. Objetivo: Realizar un diagnóstico del municipio de Castellón a través de datos facilitados por el Instituto Nacional de Estadística (INE). Metodología: Se han analizado las características de la población mayor de 60 años en cuanto a los datos demográficos, ocupación, variables que dificultan el bienestar de la población envejecida y recursos que la ciudad ofrece. Resultados: Se han establecido las características de las personas mayores en la ciudad de Castellón. Discusión: Existe la necesidad de participar en el proyecto de la oms a través de un plan de acción que proporcionará una serie de ventajas y beneficios para la ciudadanía en general y las personas mayores en particular.

Palabras clave: evaluación diagnóstica, ciudades amigables, personas mayores, proyecto OMS.

\section{Abstract}

Introduction: The population has significantly aged, as reflected by the latest World Health Organization data (WHO, 2015). An increase in the percentage of population aged 
over 60 years by the year 2050 will cause population pyramid reversal for the first time in human history. In recent years, the population aged over 60 years has migrated from rural to urban zones. By the year 2030, 3 in every 5 people worldwide are expected to live in some city. In the same vein, the number of city inhabitants in less developed regions is expected to be 4-fold higher than in developed ones. For these reasons, the city of Castellón has decided to participate in the "Global network of old population-friendly cities" project promoted by the WHO. Objective: A diagnostic study of the city of Castellón was done with Spanish National Statistics Institute (INE) data. This diagnosis takes into account the characteristics of the population aged over 60 by focusing specially on demographic information, the features that make the aged population's welfare difficult in urban centres and the resources that towns offer. Results: Our results reveal the characteristics of the aged population in Castellón. Discussion: There is an urgent need to participate in the WHO project by following an action plan to guarantee various advantages and benefits to the population in general, and to the old population of this town in particular.

Keywords: Needs Assessment, Friendly Cities, Older Persons, WHO Project.

\section{Introducción}

La población mundial ha sufrido un envejecimiento significativo según datos de la Organización Mundial de la Salud (OMS, 2015). Según los análisis estadísticos, para el año 2050 el porcentaje de población mayor de 60 años se habrá duplicado de un $11 \%$ a un $22 \%$, provocando que por primera vez en la historia la pirámide poblacional se invierta, por lo que habrá más personas mayores que niños. Si se analiza la población mundial, se observa cómo ha habido en los últimos años una migración de la población mayor de 60 años desde las zonas rurales a los centros urbanos, creándose la necesidad de adaptar las ciudades a las nuevas características de la población urbana. Para el año 2030 se prevé que tres de cada cinco personas del mundo vivan en alguna ciudad y el número de habitantes urbanos en las regiones menos desarrolladas sea casi cuatro veces mayor que en las regiones desarrolladas (OMS, 2015).

Por ello, la Organización Mundial de la Salud ha creado el proyecto de «Red mundial de ciudades amigables con las personas mayores» (OMS, 2007 a). La finalidad de este proyecto es que la población mayor, tanto en la actualidad como en el futuro, pueda disfrutar de un envejecimiento activo. La OMS define el envejecimiento activo como el proceso de optimización de las oportunidades de salud, participación y seguridad con el fin de mejorar la calidad de vida a medida que las personas envejecen (Fernández-Ballesteros, 2009; OMS, 2002). El envejecimiento activo hace referencia no solo a la capacidad física sino a la participación en cuestiones sociales, económicas, culturales y cívicas de las ciudades. Basándose en este principio de envejecimiento activo, los objetivos generales del proyecto son: generar procesos de participación comunitaria, realizar cambios en el municipio con el fin de mejorar la calidad de vida de sus ciudadanos y aprovechar el potencial que representan las personas mayores en la vida ciudadana.

La ciudad de Castellón decide participar en el proyecto para convertirse en una ciudad que haga del envejecimiento una experiencia positiva. El proyecto consta de cuatro fases: 
La fase primera es la de investigación y tiene una duración entre 1 y 2 años. Esta primera fase consta de cuatro subfases a desarrollar en un periodo de dos años:

- Fase 1.1: Diseño de mecanismos para hacer efectiva la participación de las personas mayores de la ciudad en todas las etapas del proceso, a fin de que aporten sus ideas y opiniones tanto en el diseño del proyecto como en su ejecución. Se propone así mismo la participación de otros agentes sociales, como proveedores de servicios, ciudadores informales, sectores económicos y ONG. En este punto, y por lo que se refiere a nuestra ciudad, es destacable el importante tejido asociativo con el que cuenta. En Castellón existen 30 asociaciones de personas mayores que participan activamente en la vida local. Es necesario también referirse al Consejo Local de Mayores, entidad que federa a las asociaciones de mayores de la ciudad en la defensa de los intereses que les son comunes y que mantiene un contacto frecuente y directo con todas ellas, además de ser la vía que el Ayuntamiento utiliza en numerosas ocasiones para comunicarse con las mismas. Así mismo, hay que señalar que desde la Concejalía de Gente Mayor se promueve la presencia de las personas mayores en todos los foros en los que puedan abordarse cuestiones que les afecten de manera directa.

- Fase 1.2: Evaluación inicial de la adaptación de la ciudad a las personas mayores. Esta evaluación debe incluir como mínimo cada uno de los ocho dominios que se describen en la guía de la Organización Mundial de la Salud sobre ciudades amigables con las personasm: espacios al aire libre y edificios, transportes, vivienda, participación social, respeto e integracion social, participación cívica y empleo, comunicación e información, apoyo de la comunidad y servicios de salud. Para esta evaluación se establece una metodología cualitativa a través de grupos focales, tal y como señala el Protocolo de Vancouver (OMS, 2007 b).

- Fase 1.3: Formulación de un plan de acción trienal para toda la ciudad basado en los resultados de esta evaluación. Este plan de acción debe plantearse en sintonía con todos los departamentos y entidades municipales, con la finalidad de asegurar que Castellón sea una ciudad amigable con los mayores, por lo que se convierte en una responsabilidad transversal que afecta a todas las áreas municipales.

- Fase 1.4: Establecimiento de sistemas de control. Se trata de identificar los indicadores que permitan conocer el grado de cumplimiento de las acciones propuestas.

La segunda fase es la ejecución (de 3 a 5 años de duración). Una vez completada la primera fase, y nunca más de dos años después de la adhesión a la red de la ciudad de Castellón, deberá presentarse un plan de acción para que sea examinado y aprobado por la Organización Mundial de la Salud. Una vez obtenida esta aprobación, Castellón tendrá 3 años para ejecutar su plan.

La tercera fase es la de la evaluación de los progresos (final del año 5). Al final del primer periodo de ejecución, la ciudad de Castellón deberá presentar a la Organización Mundial de la Salud un informe con los progresos realizados, que será evaluado con los indicadores definidos en la primera fase.

La cuarta fase engloba la mejora continua (ciclos de 5 años). Si resulta posible probar que se han hecho progresos con respecto al plan de acción inicial, la ciudad de Castellón pasará a una fase de mejora continua. Será invitada a elaborar un nuevo plan de acción de hasta cinco años de duración y a desarrollar los correspondientes indicadores. Los progresos con respecto a este nuevo plan se determinarán al final de este segundo periodo de ejecución. Castellón podrá seguir perteneciendo a la red mientras se comprometa a realizar nuevos ciclos de ejecución.

Mathison (2005) y Alemán y Trinidad (2012) señalan que la evaluación diagnóstica tiene como finalidad el definir y conocer las necesidades de un grupo o contexto social para hacerle frente. 
En general, una necesidad es considerada como una discrepancia entre el estado actual de un grupo de personas y su estado deseado (Altschuld y Kumar, 2010). La evalución consiste en el análisis de esas discrepancias y una posterior priorización de las acciones futuras, de tal forma que se solucionen antes las necesidades más preocupantes, básicas y urgentes (Chacón, Sanduvete, Portell y Anguera, 2013).

El objetivo de este trabajo es realizar una evaluación diagnóstica y averiguar el grado de adaptación de la ciudad de Castellón a las personas mayores.

\section{Método}

Para efectuar el diagnóstico del municipio de Castellón se ha realizado un análisis secundario de datos estadísticos procedentes tanto del Instituto Nacional de Estadística como del Ayuntamiento de Castellón. Los datos corresponden al año 2015 y los análisis se han efectuado en base a las características de la población mayor de 60 años en cuanto a los datos demográficos, vivienda, formación y ocupación, así como aquellas características que dificultan el bienestar de la población envejecida en los centros urbanos y aquellos recursos que la ciudad ofrece como ayuda a esta población.

\section{Resultados}

Los resultados del análisis indican que la población de Castellón ha envejecido entre el año 2005 y el año 2015 de forma progresiva (véase la figura 1). Las líneas verticales nos muestran la pirámide poblacional del año 2005, donde se observa cómo había más población de entre 20-30 años, mientras que actualmente hay más población entre 35 y 49.

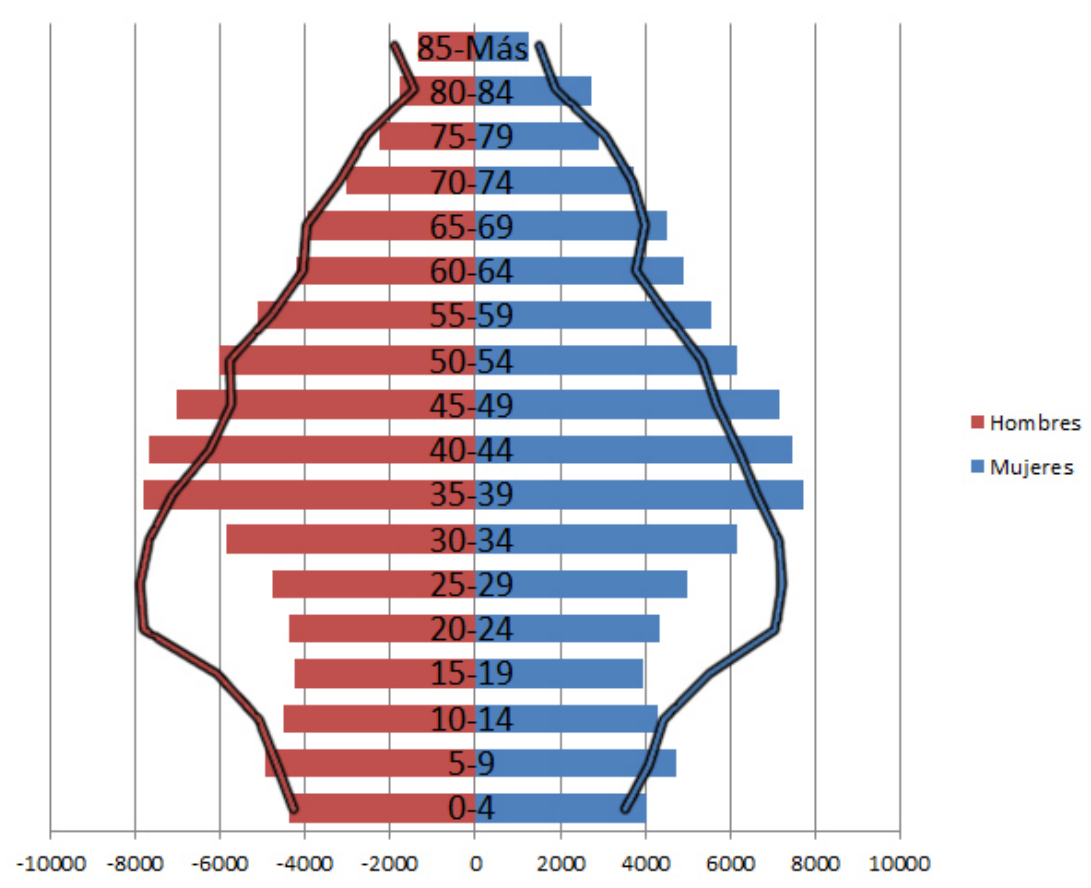

Figura 1. Pirámide poblacional de Castellón 
En la figura 2, se observa el índice de envejecimiento, esto es, el porcentaje que representa la poblacion mayor de 64 años respecto a la poblacion menor de 15 años (FernándezBallesteros, 2004). Se aprecia cómo la población mayor de 64 años se ha incrementado desde el año 2009 hasta el 2015 de forma abrupta.

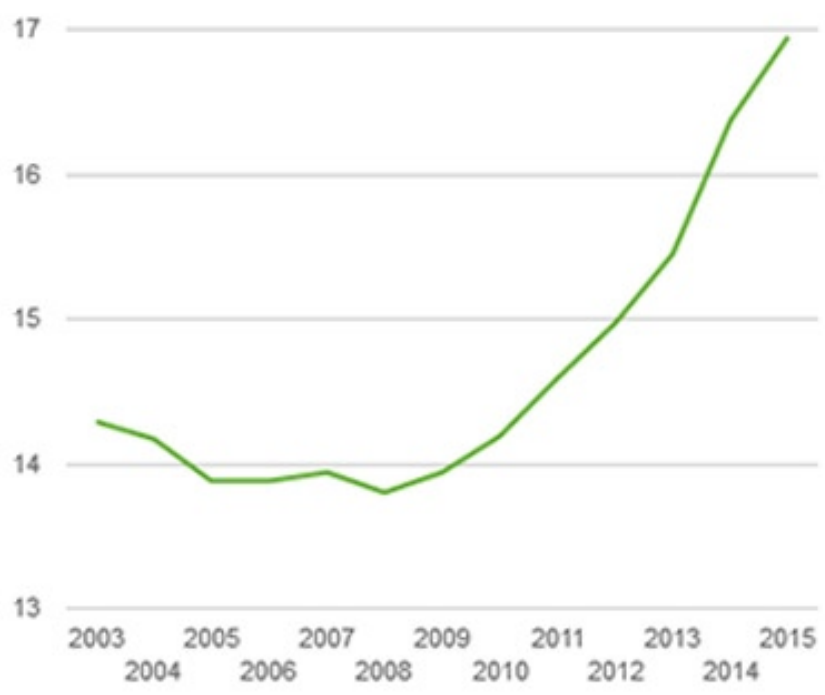

Figura 2. Índice de envejecimiento

El índice de sobreenvejecimiento representa la proporción de personas mayores de 85 o más sobre la población de 65 o más (Fernández-Ballesteros, 2004). En la figura 3 podemos observar cómo, aunque de una forma no tan abruta como el índice de envejecimiento, el índice de sobreenvejecimiento aumenta entre el 2003 y el 2015, por tanto, hay mayor población con una edad superior a 75 años.

16

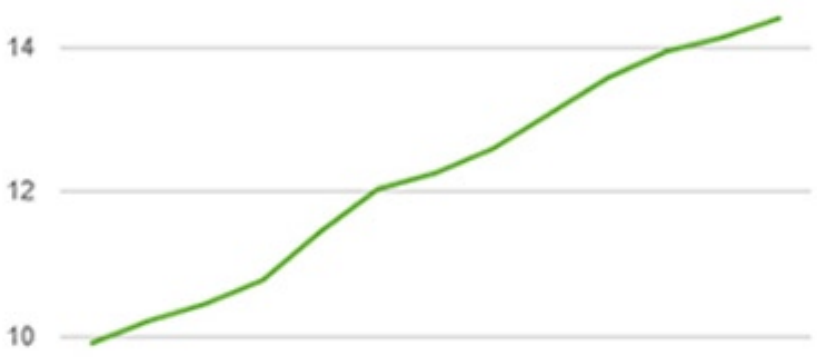

8

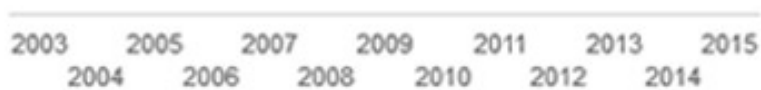

Figura 3. Índice de sobreenvejecimiento 


\section{Discusión y conclusiones}

Los resultados obtenidos muestran el envejecimiento de la población de la ciudad de Castellón, por lo que se recomienda la participación en el proyecto de la red mundial de ciudades amigables con las personas mayores; de esta forma, se podrán conocer aquellas necesidades que tienen las personas mayores de nuestro municipio, poder solventarlas y convertirlas en ventajas para las personas que en un futuro formarán parte de este grupo de población. Este proyecto proporcionará mejoras y beneficios para la ciudadanía en general y para las personas mayores en particular, permitiendo que disfruten de un envejecimiento activo que les permita envejecer con mayor dignidad y provocando cambios, reconociéndose así lo importantes que son las personas mayores, no solo como cuidadores de los nietos en vacaciones, sino como una parte de la sociedad que tiene mucho que enseñarnos.

\section{Referencias bibliográficas}

Alemán, C. y Trinidad, A. (2012). Evaluación de Servicios Sociales. Cizur Menor: Aranzadi. Altschuld, J. W. y Kumar, D. D. (2010). Needs Assessment. An Overview. Thousand Oaks: Sage.

Chacón, S., Sanduvete, S., Portell, M. y Anguera, M. T. (2013). Reporting a program evaluation: Needs, program plan, intervention, and decisions. International Journal of Clinical and Health Psychology, 13, 58-66.

Fernández-Ballesteros, R. (ed.) (2004). Gerontología Social. Madrid: Pirámide.

Fernández-Ballesteros, R. (2009). Envejecimiento activo. Contribuciones de la Psicología. Madrid: Pirámide.

Instituto Nacional de Estadística (INE) (2016). Instituto Nacional de Estadística. Recuperado el 4 de abril de 2016 de http://www.ine.es.

Mathison, S. (ed.). (2005). Encyclopedia of Evaluation. Thousand Oaks: Sage.

OMS (2002). Active Aging. A Policy Framework. Geneva: World Health Organization.

OMS (2007 a). Global Age-Friendly Cities: A Guide. Geneva: World Health Organization.

OMS (2007 b). Age-Friendly Cities Project Methodology. Vancouver Protocol. Geneva: World Health Organization.

OMS (2015). World Report on Ageing and Health. Geneva: World Health Organization. 